\title{
Significance and prognosis of epithelial-cadherin expression in invasive breast carcinoma
}

\author{
LIANG YANG, XIAO-WEN WANG, LI-PING ZHU, HONG-LI WANG, BIN WANG, \\ QIAN ZHAO and XI-YAN WANG
}

Department of Breast Surgery, Affiliated Tumor Hospital, Xinjiang Medical University, Urumqi, Xinjiang Uyghur Autonomous Region 830011, P.R. China

Received June 22, 2017; Accepted April 6, 2018

DOI: $10.3892 / \mathrm{ol} .2018 .8836$

\begin{abstract}
The objective of the present study was to investigate and analyze the epithelial-cadherin (E-cadherin) expression in invasive ductal carcinoma of the breast, and to analyze the associations between the expression and clinicopathological characteristics of lymph node metastasis and the prognosis of breast cancer. The immunohistochemical streptavidin-peroxidase method was used to detect the E-cadherin expression in 30 cases of breast fibroadenoma and in 450 cases of invasive breast cancer, and then the $\chi^{2}$ test and Kaplan-Meier method were used to analyze the data. The 30 cases of breast fibroadenoma showed positive expression of E-cadherin. Specifically, results found that E-cadherin was highly expressed in $49.04 \%$ (77/157) of patients with non-metastatic breast cancer, while low expression was found in 50.96\% (80/157). Additionally, E-cadherin was highly expressed in $29.69 \%$ (87/293) of patients with lymph node metastasis of breast cancer, with low expression in $70.31 \%$ (206/293); these differences were significantly different $\left(\chi^{2}=16.53 ; \mathrm{P}<0.001\right)$. E-cadherin was expressed in $35.48 \%$ (22/62), 33.73\% (84/249), 63.83\% (30/47) and $30.43 \%$ (28/92) of patients with luminal A type, luminal B type, human epidermal growth factor receptor-2positive and triple-negative breast cancer (TNBC), respectively. It was found that patients with high expression of E-cadherin had a better prognosis than the low expression group with regards to TNBC, and this result was significantly different $\left(\chi^{2}=4.48\right.$; $\mathrm{P}=0.034)$. In conclusion, low E-cadherin expression was associated with lymph node metastasis in invasive breast cancer, and the patients with low expression also had a poor prognosis compared with those in the high expression group. The present
\end{abstract}

Correspondence to: Dr Xi-Yan Wang, Department of Breast Surgery, Affiliated Tumor Hospital, Xinjiang Medical University, 789 East Suzhou Street, Urumqi, Xinjiang Uyghur Autonomous Region 830011, P.R. China

E-mail: tuanziqiang_qq@163.com

Key words: breast cancer, epithelial-cadherin, lymph node metastasis, molecular typing results suggested that E-cadherin could be used in a prognostic index for patients with lymph node metastasis and TNBC.

\section{Introduction}

Breast cancer is the most common cancer in females globally, with the incidence rate increasing $0.5 \%$ every year. In addition, a gradual trend is forming for the cancer to occur in females of a younger age and the morbidity rate is increasing, which severely threatens the health of women around the world $(1,2)$, although its precise mechanism is presently unclear. Growing evidence has suggested that the tumor microenvironment serves a role in the development of cancer and its prognosis $(3,4)$.

Cell adhesion molecules are a type of glycoprotein mediating cell-cell and cell-extracellular matrix adhesion, and they serve an important role in the genesis, development, invasion and metastasis of tumors (5). The decrease in tumor cell adhesion appears to easily affect tumor invasion and metastasis. Epithelial-cadherin (E-cadherin) is an important member of the cadherin family and serves an important role in the cell adhesion process. It has been reported that E-cadherin is underexpressed in a variety of tumors, and that it could participate in the growth, differentiation, metastasis and prognosis of breast cancer (6-8).

To investigate the expression of the E-cadherin protein in invasive breast cancer and its possible clinical significance, 450 cases of invasive ductal carcinoma of the breast were assessed. The expression of E-cadherin was detected using immunohistochemistry, and the association between lymph node metastasis, clinicopathological features and molecular typing was analyzed. In addition, the roles of E-cadherin in lymph node metastasis and the prognosis of breast cancer were investigated.

\section{Patients and methods}

Patients. A total of 512 patients with primary invasive ductal carcinoma of the breast were enrolled at the Affiliated Tumor Hospital of Xinjiang Medical University (Urumqi, China) between January 2001 and January 2011. The patients were randomly selected and 62 cases with non-conforming specimens were excluded from the study. All specimens were fixed using $10 \%$ formaldehyde at $36-38^{\circ} \mathrm{C}$ for $6-8 \mathrm{~h}$, embedded in paraffin and sliced into $4-\mu \mathrm{m}$ continuous pathological sections. 
Case inclusion criteria. Case inclusion criteria were as follows: Diagnosis according to breast pathology; no history of radiotherapy, chemotherapy or endocrine therapy prior to admission; Karnofsky performance status score of $\geq 80$ points and surgery performed with all samples identified by three pathology experts for histopathological identification of breast invasive ductal carcinoma; no other malignancy history; preoperative consent specimens collected and informed consent obtained; and approval provided by the Xinjiang Medical University Affiliated Cancer Hospital Ethics Committee.

Case exclusion criteria. Case exclusion criteria were as follows: Breast invasive lobular carcinoma and carcinoma in situ; and preoperative chemotherapy, radiotherapy or endocrine therapy. In addition, other exclusions were breast cancer metastases and other specific types of breast tumors (sarcoma, micropapillary carcinoma, lymphoma, inflammatory breast cancer, male breast carcinoma and gestational breast cancer).

Basic information of case data. The breast cancer patients were between 28 and 75 years of age, with a mean age of $49.30 \pm 10.48$ years, and the median age was 48 years. There were 317 premenopausal patients and 133 postmenopausal patients. All patients were female.

\section{Methods}

Immunohistochemical staining of the SP (streptavidin-peroxidase) method. Breast cancer specimens were treated with dewaxing and hydration, according to the manufacturer's protocol for immunohistochemical SP method staining (cat. no. SP0041; Beijing Solarbio Science and Technology Co., Ltd., Beijing, China), and the microwave method was used to repair antigens. Specimens of 30 benign fibroadenoma cases were obtained from patients at the Affiliated Tumor Hospital of Xinjiang Medical University, with a positive result for E-cadherin was considered as a positive control, and five fields of view were randomly selected under the microscope. Each field counted for 100 cells. Patients with benign fibroadenoma were enrolled at the Affiliated Tumor Hospital of Xinjiang Medical University between January 2001 and January 2011. The patients were randomly selected. The patients with benign fibroadenoma were between 19 and 51 years of age, and the median age was 32 years. All patients were female and there were 30 patients in the group.

Case inclusion criteria. Case inclusion criteria were as follows: Samples from surgery were observed by three pathology experts via histopathological identification to confirm fibroadenoma of the breast; Karnofsky performance status score of $\geq 80$ points; no history of tumors; and no family history of tumors.

Case exclusion criteria. Case exclusion criteria were as follows: Breast fibroadenoma with a history of breast cancer; breast fibroadenoma with a history of other malignant tumors; and breast fibroadenoma with mastitis disease.

Standard of E-cadherin staining criteria. The immunohistochemical staining results of the study were determined by three pathologists, and five x400 magnification visual fields were randomly observed. E-cadherin expression was determined by a semi-quantitative method according to the following score standard for the percentage of cell staining: $0-10 \%, 0$ points, (-); 11-25\%, 1 point, (+); 26-50\%, 2 points, (++); 51-75\%, 3 points, $(+++)$; and $>75 \%, 4$ points, $(++++)$. Those cells with a score of $\leq 2$ were considered to be negative for E-cadherin and those with a score of $\geq 3$ were considered to be positive.

Follow-up method. Telephone follow-up calls were conducted to identify associated information, the information recorded in the case was approved by the patient. Patient medical records were used to record identity, phone numbers, addresses and other personal information, including postcode, sex and history of breast cancer. Three phone calls were made to follow-up each patient, and for the patients who could not be contacted, their last discharge time was used. The follow-up calendar period was until January 31, 2016, and the follow-up time period was 1-72 months.

Tumor, Node, Metastasis staging of breast cancer was performed according to the American Joint Committee on Cancer Staging of Breast Cancer (7th Edition) (1). The histological grading of breast cancer was primarily evaluated according to the degree of glandular tube formation, nuclear polymorphisms and mitosis counts. These three aspects were scored between 3-5 points (level I; differentiation), 6-7 (level II; moderately differentiated) and 8-9 (grade III; poor differentiation) (2).

Statistical analysis. All data were shown as the mean \pm standard deviation and analyzed using a univariate analysis, $\chi^{2}$ test or exact probability method. A survival analysis was performed using the Kaplan-Meier method, with the log-rank test, and the SPSS 18.0 statistical software (SPSS, Inc., Chicago, IL, USA) was used. $\mathrm{P}<0.05$ indicated that the difference was statistically significant.

\section{Results}

Expression of E-cadherin in benign breast hyperplasia tissue. E-cadherin was mainly distributed in the gland duct epithelium and acinar epithelial cell membrane in the benign breast fibroadenoma samples, and was observed as the positive brownish-yellow granules (Fig. 1). The 30 cases of breast fibroadenoma were enrolled at the Affiliated Tumor Hospital of Xinjiang Medical University (Urumqi, China) between January 2001 and January 2011. The patients were randomly selected. The 30 cases of breast fibroadenoma were positive for E-cadherin.

Comparing the expression of E-cadherin in invasive ductal carcinoma of the breast and benign mammary gland hyperplasia. As shown in Fig. 1, E-cadherin staining was positive in breast epithelial cells and stromal cells (brownish-brown particles; SP, x400 magnification). The expression of E-cadherin in the membranes of infiltrating breast carcinoma cells is shown in Fig. 2. The positive expression rate of E-cadherin was $36.44 \%$ in the 450 cases of invasive ductal carcinoma. The high expression rate was $77 / 157$ (49.04\%) in 157 non-metastatic cancer tissues and 87/293 (29.69\%) in 293 lymph nodes with lymph node metastasis. This finding indicated that E-cadherin expression in breast cancer tissue 
Table I. Association between E-cadherin expression and lymph node metastasis.

\begin{tabular}{lccccr}
\hline & & \multicolumn{2}{c}{ Lymph node metastasis, $\mathrm{n}(\%)$} & \\
\cline { 3 - 4 } E-cadherin & $\mathrm{n}$ & Yes & No & $\chi^{2}$ & P-value \\
\hline High expression & 164 & $87(53.0)$ & $77(47.0)$ & 16.528 & $<0.001^{\mathrm{a}}$ \\
Low expression & 286 & $206(72.0)$ & $80(28.0)$ & & \\
\hline
\end{tabular}

E-cadherin -/++ is negative expression, $+++/++++$ is positive expression. Lymph node metastasis refers to the axillary lymph nodes and sentinel lymph node, and includes lymph node micrometastases, tumor metastatic lesions of $<2.0 \mathrm{~mm}$ in diameter, clusters of tumor cells and individual tumor cells. ${ }^{\mathrm{P}} \mathrm{P}<0.05$.
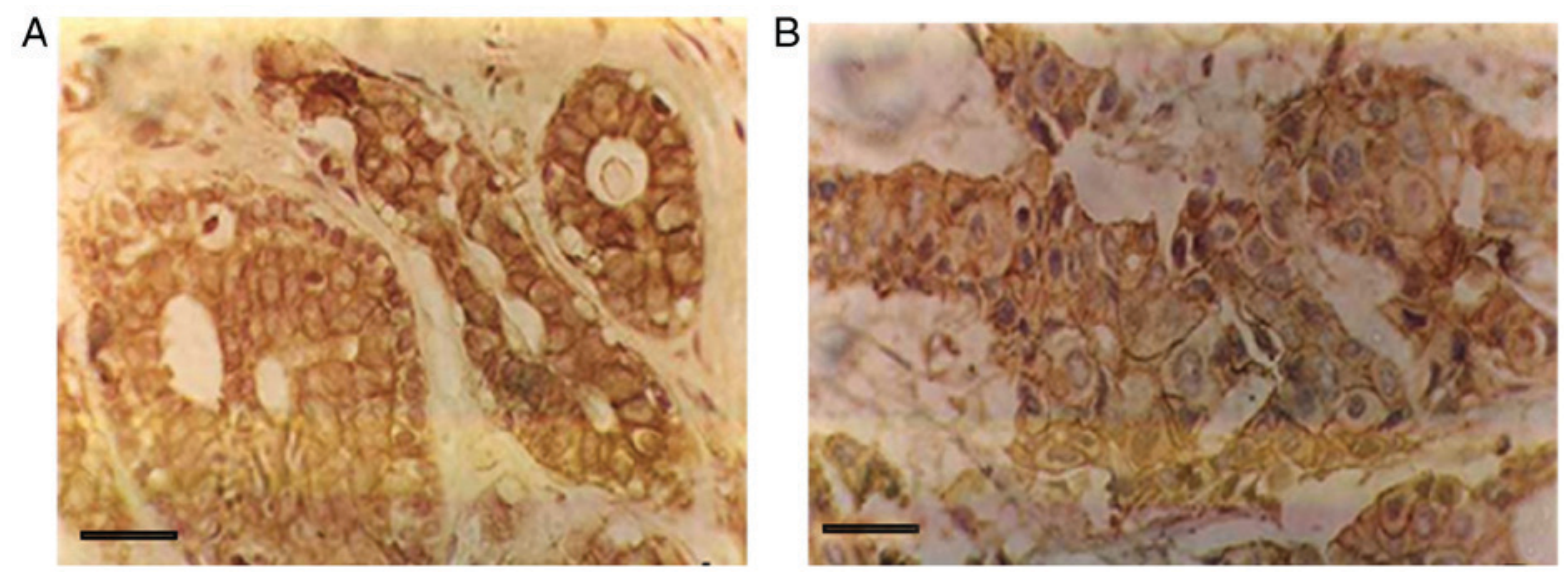

Figure 1. E-cadherin is expressed in benign and malignant breast tissues. (A) E-cadherin expression was positive in breast fibroadenoma (streptavidin peroxidase, x400 magnification). (B) E-cadherin expression was positive in breast cancer lesions (SP, x400 magnification). Scale bar, $20 \mu \mathrm{m}$.
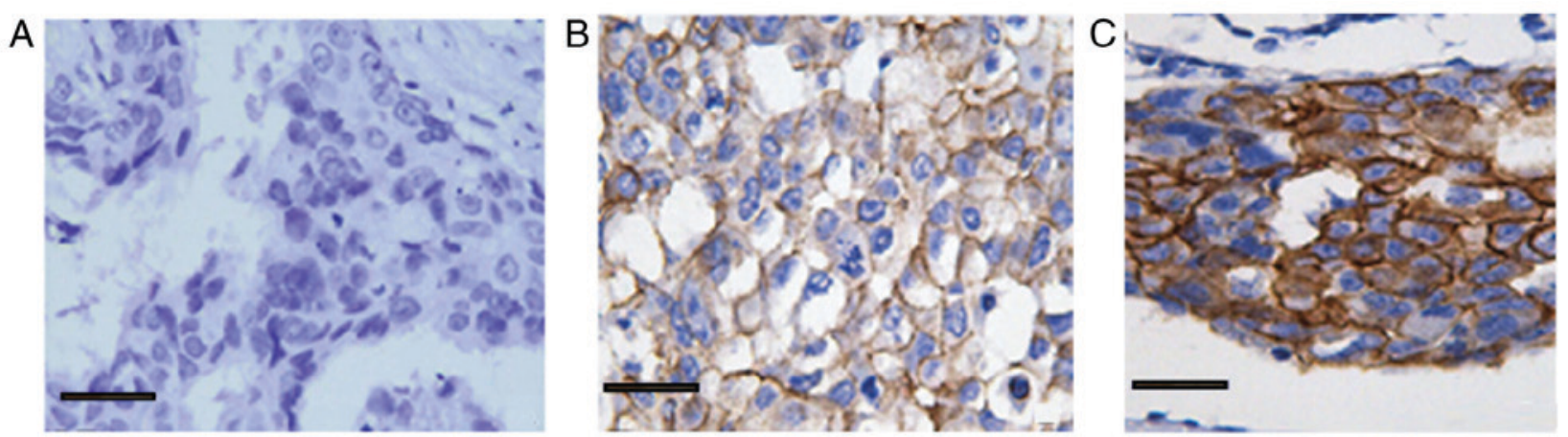

Figure 2. Expression of E-cadherin in breast cancer lesions (immunohistochemical staining, $\mathrm{x} 400$ magnification). (A) No E-cadherin expression (-). (B) Low E-cadherin expression (++). (C) High E-cadherin expression (+++). Scale bar, $20 \mu \mathrm{m}$.

is significantly lower in the lymph node metastasis of breast invasive ductal carcinoma than the breast cancer without lymph node metastasis $\left(\chi^{2}=16.528, \mathrm{P}<0.001\right.$; Table I). The low expression of E-cadherin was associated with metastatic lymph node metastasis.

Expression of E-cadherin in breast invasive ductal carcinoma and its association with the clinicopathological features of breast cancer. As presented in Table II, the expression of E-cadherin exhibited significant differences with regard to the age of the patient, lymph node metastasis, tumor size, estrogen receptor (ER) expression, molecular classification and tumor cell classification. Patients aged $>60$ years were significantly associated with the positive expression of E-cadherin. However, there were no significant differences in tumor stage, whether the patients were menopausal or premenopausal, or the expression of receptor tyrosine-protein kinase erbB-2 and proliferation marker protein $\mathrm{Ki}-67$.

Expression of E-cadherin in breast invasive ductal carcinoma and its association with the prognosis of breast cancer. As shown in Tables III and IV, and Figs. 3 and 4, survival analysis identified that there were significant differences in the expression of E-cadherin in the patients with lymph node metastasis 
Table II. Expression of E-cadherin in clinicopathological features of breast cancer.

\begin{tabular}{|c|c|c|c|c|c|c|}
\hline \multirow[b]{2}{*}{ Clinicopathological factors } & \multirow[b]{2}{*}{$\mathrm{n}$} & \multicolumn{2}{|c|}{ E-cadherin expression, $\mathrm{n}$} & \multirow{2}{*}{$\begin{array}{c}\text { High expression } \\
\text { rate, } \%\end{array}$} & \multirow[b]{2}{*}{$\chi^{2}$} & \multirow[b]{2}{*}{ P-value } \\
\hline & & High expression & Low expression & & & \\
\hline Total patients & 450 & 164 & 286 & 36.444 & & \\
\hline \multicolumn{7}{|l|}{ Age, years } \\
\hline$\leq 35$ & 87 & 40 & 47 & 45.977 & 8.373 & $0.015^{\mathrm{a}}$ \\
\hline $35-60$ & 253 & 95 & 158 & 37.549 & & \\
\hline$\geq 60$ & 110 & 29 & 81 & 26.364 & & \\
\hline \multicolumn{7}{|c|}{ Number of positive lymph nodes } \\
\hline No transfer & 157 & 77 & 80 & 49.044 & 18.796 & $<0.001^{\mathrm{a}}$ \\
\hline $1-3$ & 203 & 66 & 137 & 32.512 & & \\
\hline$\geq 4$ & 90 & 21 & 69 & 23.333 & & \\
\hline \multicolumn{7}{|l|}{ TNM staging } \\
\hline I & 65 & 20 & 45 & 30.769 & 2.162 & 0.339 \\
\hline II & 234 & 94 & 140 & 40.171 & & \\
\hline III & 151 & 54 & 97 & 35.762 & & \\
\hline \multicolumn{7}{|l|}{ Breast mass size, $\mathrm{cm}$} \\
\hline$\leq 2$ & 107 & 53 & 54 & 49.533 & 10.520 & $0.005^{\mathrm{a}}$ \\
\hline $2-5$ & 274 & 90 & 184 & 32.847 & & \\
\hline$\geq 5$ & 69 & 21 & 48 & 30.435 & & \\
\hline \multicolumn{7}{|l|}{ Menopause } \\
\hline No & 317 & 114 & 203 & 35.962 & 0.108 & 0.743 \\
\hline Yes & 133 & 50 & 83 & 37.594 & & \\
\hline \multicolumn{7}{|l|}{ c-erbB-2 } \\
\hline Positive & 85 & 25 & 60 & 29.411 & 2.238 & 0.135 \\
\hline Negative & 365 & 139 & 226 & 38.082 & & \\
\hline \multicolumn{7}{|l|}{ ER } \\
\hline Positive & 167 & 44 & 123 & 26.347 & 11.688 & $<0.001^{\mathrm{a}}$ \\
\hline Negative & 283 & 120 & 163 & 42.403 & & \\
\hline \multicolumn{7}{|l|}{$\mathrm{Ki}-67, \%$} \\
\hline$\leq 14$ & 41 & 20 & 21 & 48.780 & 2.964 & 0.085 \\
\hline$>15$ & 409 & 144 & 265 & 35.208 & & \\
\hline \multicolumn{7}{|l|}{ Molecular typing } \\
\hline Luminal A & 62 & 22 & 40 & 35.484 & 17.466 & $<0.001^{\mathrm{a}}$ \\
\hline Luminal B & 249 & 84 & 165 & 33.735 & & \\
\hline HER-2-positive & 47 & 30 & 17 & 63.830 & & \\
\hline Triple-negative & 92 & 28 & 64 & 30.435 & & \\
\hline \multicolumn{7}{|l|}{ Histological grade } \\
\hline I & 90 & 51 & 39 & 56.667 & 22.770 & $<0.001^{\mathrm{a}}$ \\
\hline II & 258 & 88 & 170 & 34.109 & & \\
\hline III & 102 & 25 & 77 & 24.510 & & \\
\hline
\end{tabular}

${ }^{\mathrm{a}} \mathrm{P}<0.05$. TNM, tumor-node-metastasis; c-erb-B2, receptor tyrosine-protein kinase erbB-2; ER, estrogen receptor; Ki-67, proliferation marker protein Ki-67; HER-2, human epidermal growth factor receptor 2.

$\left(\chi^{2}=9.546, \mathrm{P}=0.002\right)$ and in those with $\mathrm{TNBC}\left(\chi^{2}=4.48\right.$, $\mathrm{P}=0.03)$. The low expression of E-cadherin inpatients with TNBC is associated with poorer prognosis. There was no significant difference in the prognosis of the untreated lymph node group, or the Luminal A, Luminal B or human epidermal growth factor receptor 2 (HER-2)-positive group.

\section{Discussion}

The incidence of breast cancer continues to grow, and it is currently the most common form of malignant tumor among Chinese women (3). Breast cancer metastasis seriously affects the prognosis of patients and is the leading cause of 
Table III. Expression of E-cadherin in lymph node metastasis and non-metastasis of breast cancer was analyzed by statistical analysis (Kaplan-Meier method).

\begin{tabular}{|c|c|c|c|c|c|c|}
\hline \multirow[b]{2}{*}{ Test statistic } & \multicolumn{3}{|c|}{$\begin{array}{l}\text { Non-breast lymph } \\
\text { node metastasis group }\end{array}$} & \multicolumn{3}{|c|}{$\begin{array}{l}\text { Breast cancer lymph } \\
\text { node metastasis group }\end{array}$} \\
\hline & df & $\chi^{2}$ & P-value & df & $\chi^{2}$ & P-value \\
\hline Log-rank (Mantel-Cox) & 1 & 3.628 & 0.057 & 1 & 9.546 & $0.002^{\mathrm{a}}$ \\
\hline Breslow (generalized Wilcoxon) & 1 & 3.191 & 0.074 & 1 & 12.234 & $<0.001^{\mathrm{a}}$ \\
\hline Tarone-Ware & 1 & 3.417 & 0.065 & 1 & 10.862 & $0.001^{\mathrm{a}}$ \\
\hline
\end{tabular}

${ }^{a} \mathrm{P}<0.05$. There were no statistically significant differences in the three survival statistic tests in the lymph node metastasis group. There was a significant difference in E-cadherin expression and low expression survival analysis in the lymph node metastasis group. df, degrees of freedom.

Table IV. Expression of E-cadherin in different molecular types of breast cancer: Survival analysis (Kaplan-Meier method).

\begin{tabular}{|c|c|c|c|c|c|c|c|c|c|}
\hline \multirow[b]{2}{*}{ Test statistic } & \multirow[b]{2}{*}{ df } & \multicolumn{2}{|c|}{ Luminal A } & \multicolumn{2}{|c|}{ Luminal B } & \multicolumn{2}{|c|}{ HER-2-positive } & \multicolumn{2}{|c|}{ Triple-negative } \\
\hline & & $\chi^{2}$ & $\mathrm{P}$-value & $\chi^{2}$ & P-value & $\chi^{2}$ & P-value & $\chi^{2}$ & P-value \\
\hline Log-rank (Mantel-Cox) & 1 & 2.87 & 0.09 & 4.33 & 0.04 & 0.61 & 0.43 & 4.48 & $0.03^{\mathrm{a}}$ \\
\hline Breslow (generalized Wilcoxon) & 1 & 2.87 & 0.09 & 3.31 & 0.07 & 0.51 & 0.48 & 5.42 & $0.02^{\mathrm{a}}$ \\
\hline Tarone-Ware & 1 & 2.87 & 0.09 & 3.75 & 0.05 & 0.51 & 0.46 & 5.03 & $0.02^{\mathrm{a}}$ \\
\hline
\end{tabular}

${ }^{\mathrm{a}} \mathrm{P}<0.05$. df, degrees of freedom; HER-2, human epidermal growth factor receptor 2.

Expression of E-cadherin and prognosis of patients with A breast cancer and non-metastatic lymph nodes

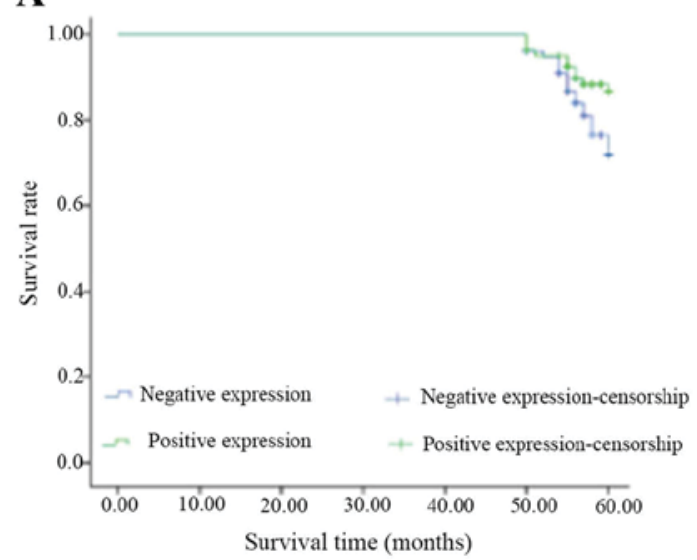

Expression of E-cadherin and prognosis of patients with
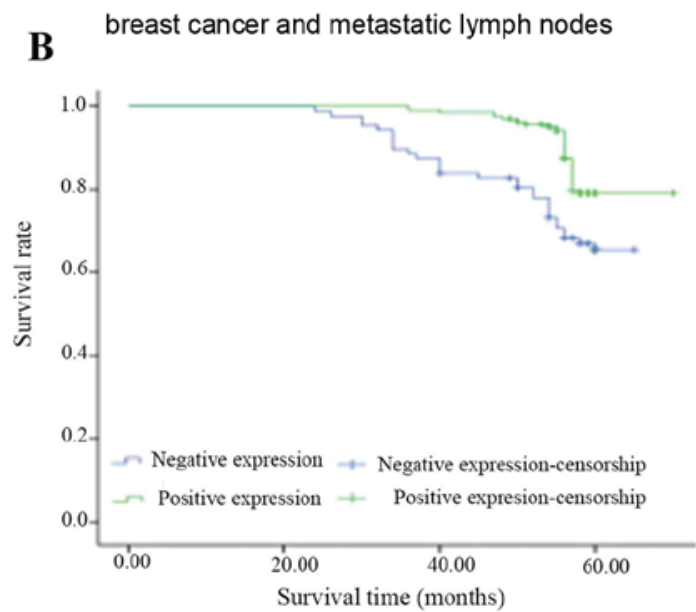

Figure 3. Expression of E-cadherin and patient prognosis in breast cancer with non-metastatic lymph nodes. (A) Non-breast lymph node metastasis group ( $n=157$ cases). (B) Breast cancer lymph node metastasis group ( $n=293$ cases).

mortality (4). Of those patients who receive an early diagnosis of breast cancer subsequent to receiving adjuvant therapy, $\sim 30 \%$ will eventually develop recurrence or metastasis $(5,6)$. Breast cancer recurrence and metastasis are severe clinical problems.

Changes in cell adhesion are the main mechanism of invasion and metastasis of a malignant tumor. Changes in adhesion molecules may reduce the adhesion of tumor cells, contributing to tumor infiltration and metastasis. Therefore, the decline of cell adhesion is an important factor leading to tumor metastasis (7).

E-cadherin is a type of cell adhesion glycoprotein; it is not only a tumor cell invasion and metastasis inhibitor, but it is also a normal cell growth inhibitor. Studies have found that when epithelial-mesenchymal transition occurs in cancer cells, E-cadherin expression decreases or shows a functional loss, thus causing decreased cell adhesion, loss of polarity and infiltration of the surrounding tissue growth, and it may be 
Expression of E-cadherin and prognosis

of patients with Luminal A-type breast cancer

A

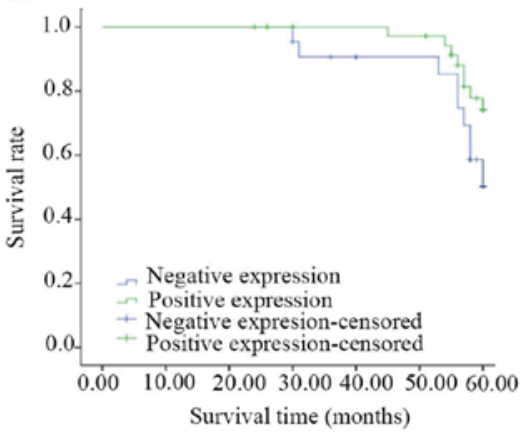

Expression of E-cadherin and prognosis

C of patients with HER-2-positive breast cancer

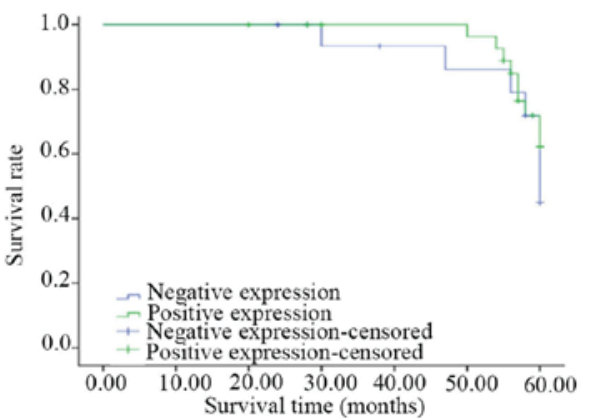

Expression of E-cadherin and prognosis

of patients with Luminal B-type breast cancer

B

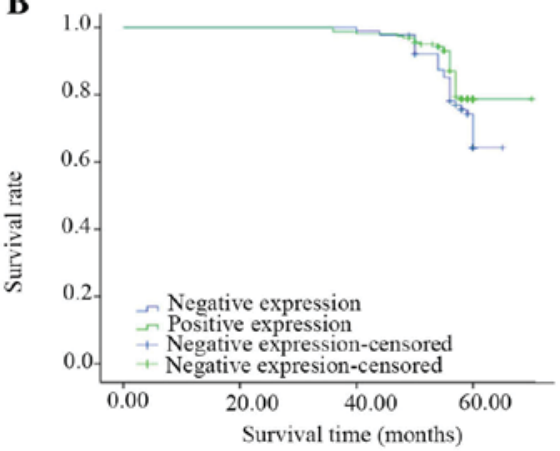

Expression of E-cadherin and prognosis

D of patients with triple-negative breast cancer

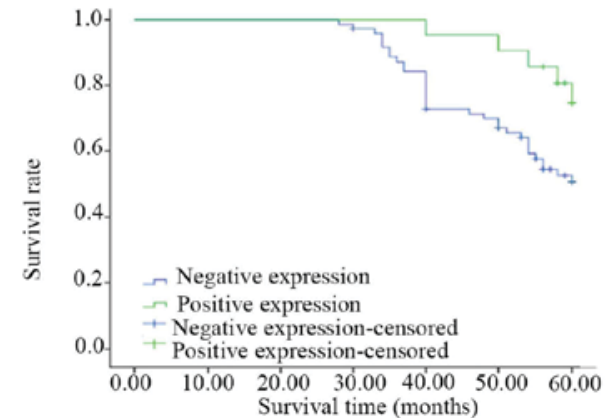

Figure 4. Expression of E-cadherin and prognosis of breast cancer patients. (A) Luminal A-type breast cancer group (n=62 cases). (B) Luminal B-type breast cancer group ( $\mathrm{n}=249$ cases). (C) Human epidermal growth factor receptor-2-positive breast cancer group ( $\mathrm{n}=47$ cases). (D) Triple-negative breast cancer group $(\mathrm{n}=92$ cases $)$

transferred to bone, liver, lung and brain tissue (8). E-cadherin has become one of the research hotspots among the cadherin family members. Studies have found that E-cadherin is involved in the early occurrence, infiltration and metastasis of different tumors (9-12). The expression is of E-cadherin is closely associated with the invasion and metastasis of a number of tumors and their clinical prognoses (13-15).

The present study results suggested that low expression or deletion of E-cadherin was positively associated with lymph node metastasis. The negative expression rate was significantly higher in breast cancer patients with local lymph node metastasis than in patients without local lymph node metastasis, and the differences were statistically significant. Presumably, under normal circumstances, E-cadherin serves a role in maintaining cell morphology within the body (16). The present findings further suggested that E-cadherin was closely associated with the infiltration and metastasis of breast ductal carcinoma and could be used as a marker to predict the lymph node metastasis of invasive ductal carcinoma. The study results suggested that E-cadherin expression was associated with the prognosis of patients with breast cancer. The 5-year survival rate was higher in the E-cadherin-positive group than that in the E-cadherin-negative group in lymph node metastatic invasive ductal carcinoma, and the differences were statistically significant $\left(\chi^{2}=16.53, \mathrm{P}<0.001\right)$. E-cadherin expression was associated with the molecular typing of breast cancer. E-cadherin exhibited low expression in breast cancer and TNBC, which was closely associated with the invasion and metastasis of TNBC. The expression of E-cadherin in HER-2-negative and ER-positive samples was high, indicating that ER-positive expression may be involved in the regulation of E-cadherin expression.

Understanding these mechanisms and further investigating the findings of this study will aid in confirming these results and determining other associated important findings. Further research is required to adequately understand the decrease in cell adhesion.

\section{Acknowledgements}

Not applicable.

\section{Funding}

This study was supported by funds from the Xinjiang Uygur Autonomous Region Natural Science Foundation (grant no. 2016D01C353).

\section{Availability of data and materials}

The datasets used and/or analyzed during the current study are available from the corresponding author on reasonable request.

\section{Authors' contributions}

XYW designed the experiment; LY, LPZ, BW and QZ performed the experiments; XWW collected, analyzed and 
interpreted data; LY drafted the manuscript and revised it critically for important intellectual content, giving final approval of the version to be published; HLW performed analysis and interpretation of data, and modified the paper.

\section{Ethics approval and consent to participate}

All subjects provided written informed consent. The present study was approved by the Ethics Committee of the Affiliated Tumor Hospital, Xinjiang Medical University (Urumqi, China).

\section{Consent for publication}

All patients agreed to the publishing of the data.

\section{Competing interests}

The authors declare that they have no competing interests.

\section{References}

1. Cabioglu N: Staging of Breast Cancer. In: Breast Disease. Springer International Publishing, Basel, p1360, 2016

2. Elston CW and Ellis IO: Pathological prognostic factors in breast cancer. I. The value of histological grade in breast cancer: Experience from a large study with long-term follow-up. C. W. Elston \& I. O. Ellis. Histopathology 1991; 19; 403-410. Histopathology 41: 403-410, 2002.

3. Miller KD, Siegel RL, Lin CC, Mariotto AB, Kramer JL, Rowland JH, Stein KD, Alteri R and Jemal A: Cancer treatment and survivorship statistics, 2016. CA Cancer J Clin 66: 271-289, 2016.

4. Chen W, Zheng R, Baade PD, Zhang S, Zeng H, Bray F, Jemal A, Yu XQ and He J: Cancer statistics in China 2015. CA Cancer J Clin 66: 115-132, 2016.

5. Milioli HH, Santos Sousa K, Kaviski R, Dos Santos Oliveira NC, De Andrade Urban C, De Lima RS, Cavalli IJ and De Souza Fonseca Ribeiro EM: Comparative proteomics of primary breast carcinomas and lymph node mastases outlining markers of tumor invasion. Cancer Genomics Proteomics 12: 89-101, 2015.
6. Berman AT, Thukral AD, Hwang WT, Solin LJ and Vapiwala N: Incidence and patterns of distant metastases for patients with early-stage breast cancer after breast conservation treatment. Clin Breast Cancer 13: 88-94, 2013.

7. Li DM and Feng YM: Signaling mechanism of cell adhesion molecules in breast cancer metastasis: Potential therapeutic targets. Breast Cancer Res Treat 128: 7-21, 2011.

8. Paredes J, Figueiredo J, Albergaria A, Oliveira P, Carvalho J, Ribeiro AS, Caldeira J, Costa AM, Simões-Correia J, Oliveira MJ, et al: Epithelial E-and P-cadherins: Role and clinical significance in cancer. Biochim Biophys Acta 1826: 297-311, 2012.

9. Ye Y, Tian H, Lange AR, Yearsley K, Robertson FM and Barsky SH: The genesis and unique properties of the lymphovascular tumor embolus are because of calpain-regulated proteolysis of E-cadherin. Oncogene 32: 1702-1713, 2013.

10. Xue $\mathrm{S}$ and Chen YX: Progression on the roles of E-cadherin in invasion and metastasis of gastric cancer. Chin Clin Oncol 6: 555-558, 2015 (In Chinese).

11. Tian MY, Wang LH and Zhang X: Expressions of E-cadherinand Vimentin in Lung Cancer Tissueand Their Relationship to Epithelial-Mesenchymal Transition. Chin J Biol 24: 1068-1071, 2011 (In Chinese).

12. Wendt MK, Taylor MA, Schiemann BJ and Schiemann WP: Down-regulation of epithelial cadherin is required to initiate metastatic outgrowth of breastcancer. Mol Biol Cell 22: 2423-2435, 2011.

13. Wang YM, Wang YC, Wang RF, Wang LG and Tao N: Expressions of MicroRNA-221 and E-cadherin in gastric cancer and their relationship with clinical significance. Progress in Modern Biomedicine 31: 6088-6091, 2017 (In Chinese).

14. Iseki Y, Shibutani M, Maeda K, Nagahara H, Ikeya T and Hirakawa K: Significance of E-cadherin and CD44 expression in patients with unresectable metastatic colorectal cancer. Oncol Lett 14: 1025-1034, 2017.

15. Spachmann PJ, Otto W, Vergho D, Kalogirou C, Prohaska S, Weber F, Evert M, Burger M, Denzinger S, Kübler H and Breyer J: High expression of E-cadherin and $\beta$-catenin is associated with development of metastases and predicts worse survival in renal cell carcinoma with invasion of the vena cava. European Urology Supplements 16: e2846, 2017.

16. Humar B and Guilford P: Hereditary diffuse gastric cancer: A manifestation of lost cell polarity. Cancer Sci 100: 1151-1157, 2009. 